\section{Plastic optical fiber sensor for measuring driver-gripping force}

\author{
Jaehee Park \\ Keimyung University \\ Department of Electronic Engineering \\ 1000, Sindang-Dong \\ Dalseo-Gu, Daegu, 704-701, Republic of Korea \\ E-mail: jpark@kmu.ac.kr
}

\begin{abstract}
This study presents a plastic optical fiber (POF) sensor for measuring the driver's gripping force applied to a steering wheel. This sensor consists of a POF wound around the steering wheel covered with a soft material. The sensing method is based on the bending radiation loss of the POF induced by the driver's gripping force applied to the steering wheel. The bending radiation loss is proportional to the gripping force, and the response time of the POF sensor is about $2 \mathrm{~s}$. The experimental results show that a POF sensor can be effective for monitoring the driver gripping force, and hence driver fatigue. (C) 2011 Society of Photo-Optical Instrumentation Engineers (SPIE). [DOI: 10.1117/1.3542040]
\end{abstract}

Subject terms: plastic optical fiber sensor; gripping force; bending loss; safety driving.

Paper 100874LR received Oct. 26, 2010; revised manuscript received Dec. 21, 2010; accepted for publication Dec. 27, 2010; published online Feb. 4, 2011.

A major cause of vehicle accidents is a driver with a diminished vigilance level due to fatigue. In this case, the driver experiences a marked decline in their perception, recognition, and ability to control the vehicle, thereby seriously endangering their own life and the lives of others. Therefore, much effort has already been focused on developing methods for detecting driver fatigue, including monitoring the eye movements, ${ }^{1,2}$ brain waves, ${ }^{3}$ or heart rate variability. ${ }^{4}$ Yet, while some good results have been reported, none of these methods is very practical. Thus, recent interest has focused on monitoring the driver's gripping force, which is a physiological parameter associated with driver fatigue.

Commonly used gripping-force sensors include piezoresistive sensors and piezoelectric sensors, ${ }^{5}$ which have already been widely applied in the areas of robotics and industrial automation, bio-medicine, entertainment, and food processing. However, these sensors are not adequate for measuring the gripping force of a driver due to their poor performance and limited capacity in a vehicle setting. ${ }^{6}$ Therefore, in place of these sensors, microbend sensors ${ }^{7,8}$ that use the glass optical fibers have been developed for measuring physical variables, such as pressure, strain, and gripping forces. Yet, these sensors are too costly for practical implementation in vehicles, due to the difficulty involved in handling glass fibers. As an alternative, easy handling plastic optical fiber (POF) sensors ${ }^{9,10}$ are increasingly being used for sensing physical parameters in vehicles. Therefore, this paper investigates the

0091-3286/2011/\$25.00 @ 2011 SPIE ability of POF sensors to measure the gripping force of a driver.

The gripping force sensor for monitoring driver fatigue consists of a POF wound around the steering wheel covered by a soft material. The sensing method is then based on the radiation loss due to the bending of the POF induced by the driver's gripping force. The bending radiation loss of the POF can be explained based on a geometric optics method. When light rays pass through a bent section of a step-index POF, radiation loss is caused due to a change of the incident angle at the core-cladding interface. If the incident angle of the light ray is larger than a critical angle, the light ray reflects back into the core. Otherwise, some part of the ray is tunneled into the cladding and the remainder is bounced back into the core. As such, the ray power loss $\left(P_{l}\right)^{11}$ due to tunneling at the interface can be calculated from:

$P_{l}=\frac{4 \cos \alpha \sqrt{\left(\cos ^{2} \alpha-\cos ^{2} \alpha_{c}\right)}}{\left[\cos \alpha+\sqrt{\left(\cos ^{2} \alpha-\cos \alpha_{c}\right)}\right]^{2}} * P_{i}$

where $P_{i}$ is the power of the incident ray, $\alpha$ is the incident angle with the normal to the interface, and $\alpha_{c}$ is the critical angle. In a previous theoretical analysis ${ }^{11}$ based on the assumption that the light power flows along the core within tubes of parallel rays in an infinitesimal cross-section of a step-index POF, the results indicated that the bending loss was inversely proportional to the bending radius and was a function of the refractive index of the core and cladding. Thus, when a driver grips the steering wheel, the gripping force induces bending of the POF wound around the steering wheel. This bending results in the radiation loss of light traveling inside the POF. Since a stronger gripping force creates a smaller bending radius, the radiation loss is proportional to the gripping force. Therefore, the driver's gripping force can be measured from the radiation loss of light propagating inside the POF.

A diagram of the experimental configuration used to measure the gripping force is shown in Fig. 1. The experimental setup consisted of a $650 \mathrm{~nm}$ laser diode (LD), a steering wheel, a 5-mm thick polyethylene wheel cover, stepindex POF (Twestech PGR-FB-1000) acting as the gripping force sensor, optical power meter (Thorlabs PM100), personal computer (PC), two voltage meters, interface electronic circuits, and two tactile sensors (FlexForce A201-100) using piezoresistive materials. A POF 56-m in length with an outer diameter of $1 \mathrm{~mm}$ (a core diameter of $0.98 \mathrm{~mm}$ and cladding thickness of $0.02 \mathrm{~mm}$ ) was wound around part of the polyethylene wheel cover which covered the steering wheel. The bending diameter of the POF was about $42 \mathrm{~mm}$ when wound around the wheel cover. The refractive index of the core and cladding were 1.49 and 1.41 , respectively. Two tactile sensors were also placed at location A and B just under the POF to measure the gripping force applied to the steering wheel. An optical power meter was used to measure the light power that reached the end of the POF and two voltage meters and interface circuits were used to measure the output resistances of two tactile sensors according to the applied gripping force. The light emitting from a LD was coupled into the POF wound around the wheel cover and traveled inside the POF. When the light arrived at the end of the POF, the light was converted to an electric voltage using the optical power meter. When the driver grasped the steering wheel, a gripping force was generated in the area where the 


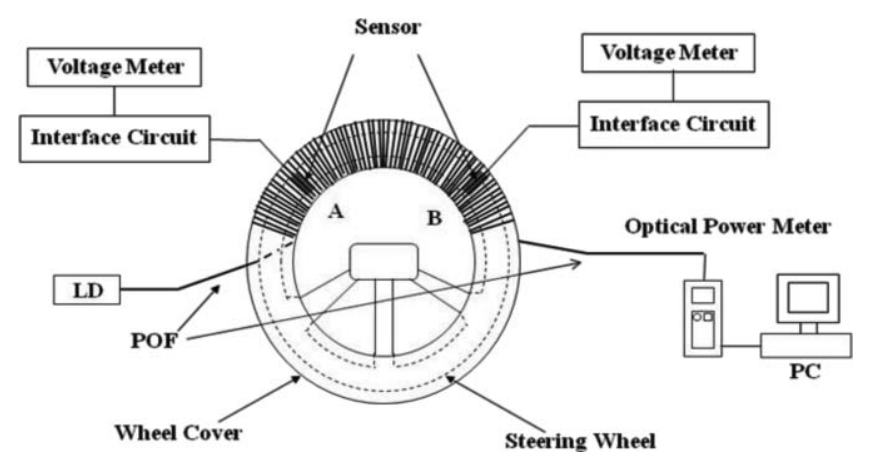

Fig. 1 Experimental setup.

steering wheel was being held. This gripping force then induced bending of the POF wound around the parts of the steering wheel being held, and the bending created a radiation loss of the light passing through the held area. Thus, based on the power of the light that reached the end of the POF, the gripping force applied to the steering wheel was measured. The gripping force was generated by pressing a $10-\mathrm{cm}$ length wooden board on the POF sensor by hand. The amplitude of the applied gripping force was obtained from the output voltage of the interface circuit connected to the tactile sensor.

The power loss generated by winding the POF around the wheel cover was measured first. Before winding, the light power measured at the end of the POF was about $100 \mathrm{uW}$, and after winding, it was about $70 \mathrm{uW}$, indicating a power loss of about $30 \mathrm{uW}$, which was due to the intrinsic transmission loss of the POF and tight winding of the POF around the steering wheel cover. After checking the power loss due to the winding, the power of the light detected at the end of the POF was measured when the gripping force was applied to the steering wheel. Figure 2 [lines (a) and (b)] show the light power detected according to the gripping force applied to location $\mathrm{A}$ of the steering wheel and location B, respectively. The detected power decreased as the gripping force increased, as in the theoretical analysis results, and the relationship between the detected light power and the gripping force was approximately linear when the applied force was over $17.64 \mathrm{~N}$. In the linear region, the slope of Fig. 2 [line (a)] was $0.319 \mathrm{uW} / \mathrm{N}$, while the slope of Fig. 2 [line (b)] was $0.294 \mathrm{uW} / \mathrm{N}$. Although the same gripping force was applied to both locations, the radiation loss at location A was slightly higher than that at location $\mathrm{B}$, as the power of the light passing through location $\mathrm{A}$ was higher than that passing through location $\mathrm{B}$ when the driver did not hold the wheel. Figure 2 [line (c)] shows the optical power variations detected when the same amount of gripping force was applied at locations A and B simultaneously. In this case, the radiation loss was less than the sum of the radiation losses produced when the gripping force was applied at location A or B separately, as when the gripping force was applied to both locations, the power of the light passing through location B was lower than that when the gripping force was only applied at location B. The optical power variations (Fig. 3) induced by variations in the applied gripping force over time were displayed on the PC using the LABVIEW program given by Thorlabs. A response time was about $2 \mathrm{~s}$, enough to monitor a change in the gripping force of the driver in a vehicle on the road. If a harder material is used for the wheel cover, a faster response time can be expected. The outputs of the tactile reference sensors used in the experiments

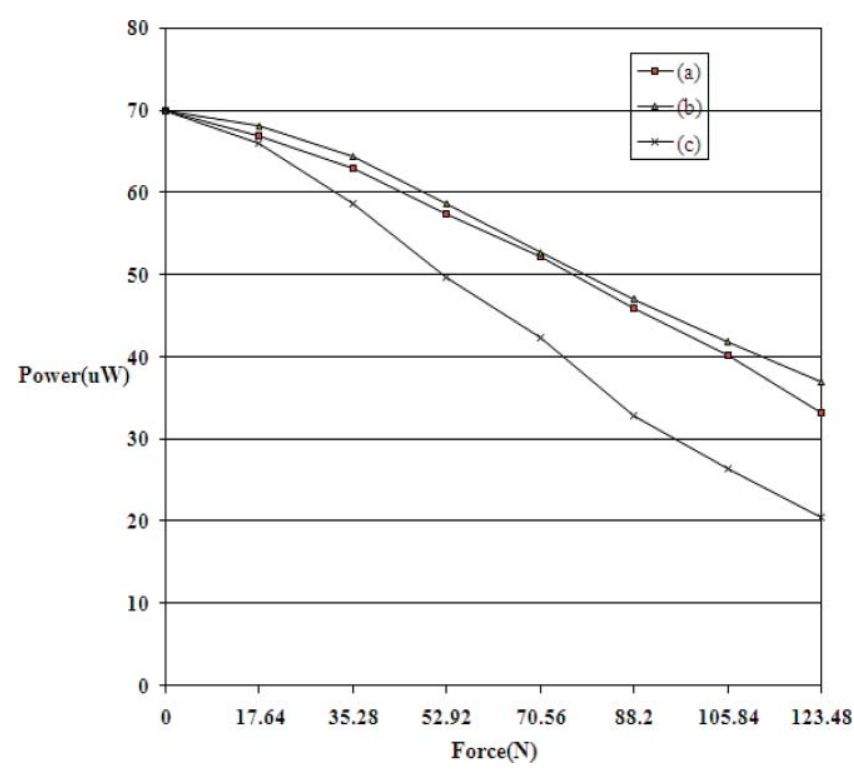

Fig. 2 Optical power detected at optical power meter as a function of the gripping force applied to the steering wheel.

change due to the thermally induced stress. For more accurate measurements, the temperature controlled tactile sensors have to be utilized as reference sensors. Additionally, the influence of a driver's hand size for precise measurements has to be investigated. When a driver is in a drowsy state, this induces a lower gripping force, resulting in the detection of a higher light power. Therefore, drowsy driving can be detected from the light power measured using a POF gripping force sensor.

This paper investigates the use of a POF sensor wound around a steering wheel for measuring the gripping force of a driver. The measurement method is based on the bending radiation loss of the POF induced by the driver's gripping force applied to the steering wheel. The radiation loss was found to be proportional to the gripping force applied to the steering wheel, and the sensor response time was about 2 s. A faster response time can be anticipated if a harder material than polyethylene is used for the wheel cover and more precise measurements can be expected if temperature controlled tactile sensors are used for calibration. The experimental results show that winding a POF sensor wound around a steering wheel can be used to measure the gripping force related to driver fatigue. Further study will include the influence of a driver's hand size and testing the POF sensor

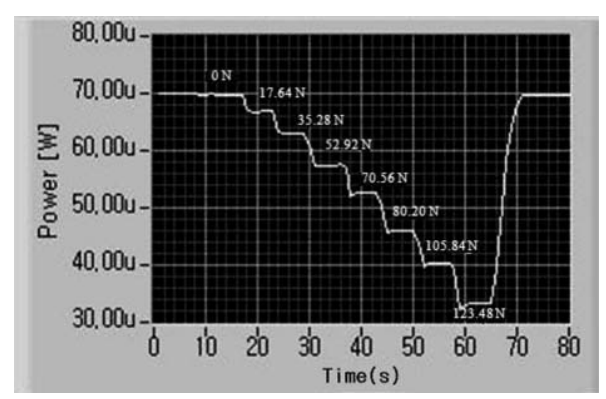

Fig. 3 Optical power variations when varying applied gripping force over time. 


\section{OE Letters}

gripping force measurements using a vehicle on the road, and will also examine the relationship between driver fatigue and the POF sensor gripping force measurements.

\section{Acknowledgments}

The present research has been conducted by the Bisa Research Grant of Keimyung University in 2006.

\section{References}

1. Q. Ji, Z. Zhu, and P. Lan, "Real-time nonintrusive monitoring and prediction of drive fatigue," IEEE Trans. Veh. Technol. 53, 1052-1068 (2004).

2. M. Eriksson and N. Papanikolopoulos, "Driver fatigue: a vision-based approach to automatic diagnosis," Transp. Res., Part C: Emerg. Technol. 9, 399-413 (2001).
3. S. Lai and A. Craig, "A critical review of the phychophysiology of driver fatigue," Biol. Psychol. 55, 173-194 (2001).

4. S. Eom, J. Park, and J. Lee, "Optical fiber arterial pulse wave sensor," Microwave Opt. Technol. Lett. 52, 1318-1321 (2010).

5. G. Krishna and K. Rajanna, "Tactile sensor based on piezoelectric resonance,” IEEE Sens. J. 4, 691-697 (2004).

6. Y. Lin, H. Leng, G. Yang, and H. Cai, "An intelligent noninvasive sensor for driver pulse wave measurement," IEEE Sens. J. 7, 790-799 (2007)

7. J. Berthold, "Historical review of microbend fiber-optic sensors," $J$ Lightwave Technol. 7, 1193-1199 (1995).

8. D. Lee, K. Kwon, J. Song, and J. Park, "Fabrication and analysis of microbend fiber sensor using bending-sensitivity fiber," Opt. Eng. 45 014401.1-014401.9 (2006).

9. B. Beadle, R. Weis, and C. Norwood, "Radial strain-induced attenuation in plastic optical fiber," Opt. Eng. 35, 1696-1699 (1996).

10. M. Linec and D. Donlagic, "A plastic optical fiber microbend sensor used as a low-cost anti-squeeze detector," IEEE Sens. J. 7, 1262-1267 (2007).

11. J. Arrue, J. Zubia, G. Fuster, and D. Kalymnios, "Light power behavior when bending plastic optical fibres," IEE Proc. Optoelectron. 145, 313 318 (1998). 